\title{
On calibrated weights in stratified sampling $\begin{array}{lll}\text { D. K. Rao } & \text { M. G. M. Khan } & \text { G. K. Singh } \\ & & \end{array}$
}

Received 19 November 2017; Revised 04 April 2018

\begin{abstract}
In this paper, we propose a calibration estimator of population mean in stratified sampling using the known mean and variance information from multi-auxiliary variables. The problem of determining the optimum calibrated weights is formulated as an optimisation problem and is solved using the Lagrange multiplier technique. A numerical example with real data is presented to illustrate the computational details of the proposed estimator. A comparison study is also carried out using real and simulated data to evaluate the performance and the usefulness of the proposed estimator. The study reveals that the proposed estimator with multi-auxiliary information is the most efficient estimator of the population mean when compared to other estimators as it provides least estimated variance and highest gain in relative efficiency (RE).
\end{abstract}

Subject class: 62D05; 62-02

DOI:10.21914/anziamj.v59i0.12668, C) Austral. Mathematical Soc. 2018. Published September 24, 2018, as part of the Proceedings of the 13th Biennial Engineering Mathematics and Applications Conference. ISSN 1445-8810. (Print two pages per sheet of paper.) Copies of this article must not be made otherwise available on the internet; instead link directly to the DOI for this article. 
Keywords: Stratified sampling; calibration estimation; auxiliary information

\section{Contents}

1 Introduction

C191

2 The Problem of Calibrated Weights

3 Determining the Optimum Calibrated Weights

C195

4 Numerical Illustration and Comparison Study

C196

4.1 Numerical Illustration . . . . . . . . . . . . . . .

C196

4.2 Comparison Study . . . . . . . . . . . . .

5 Conclusion

C201

References

C202

\section{Introduction}

Calibration estimation, on which the current research is conducted, dates back to 1992. A large amount of literature is being devoted to it, gaining significant attention in the field of survey methodology and survey practice. It is a technique that uses available auxiliary information to improve the precision of the survey estimates. The technique works by minimising the chi-square distance function subject to some calibration constraints. The notion of calibration estimators was first introduced by [1] in survey sampling.

Since then several survey statisticians have contributed to the study of calibrated estimation in survey sampling $[2,3,6,10,13,11,12,15]$. Singh et al. [10] introduced the calibration approach in stratified random sampling 
where they proposed the combined generalised regression (GREG) estimator of population mean using the known mean information from a single auxiliary variable. Later, many authors have contributed to the theory of calibration estimation in stratified sampling $[5,8,7,11,9,14]$.

The purpose of this paper is to propose a calibration estimator of population mean in stratified sampling using the known mean and variance information from several auxiliary variables. Our main contributions include (1) introducing new calibration constraints; (2) generalising the problem with multi-auxiliary variables; (3) investigating the efficiency of the proposed estimators; and (4) investigating whether the information from several auxiliary variables improves the estimate of population mean.

The problem of determining the optimum calibrated weights is formulated as an optimisation problem that minimises the chi-square type distance, subject to some new calibration constraints. The problem is then solved to determine the calibrated weights using the Lagrange multiplier technique. The computational details of the procedure are illustrated in the presence of two auxiliary variables. A numerical example with real data is presented to demonstrate the computational details of the proposed estimator. To compare the efficiency gain of the proposed multivariate estimator with the other calibration estimators a comparison study is carried out. The study reveals that the proposed multivariate estimator is more efficient than the other calibration estimators.

\section{The Problem of Calibrated Weights}

Consider that a finite population $\mathrm{U}=\{1,2, \ldots, \boldsymbol{i}, \ldots, \mathbf{N}\}$ of size $\mathbf{N}$ is stratified into $\mathrm{L}$ strata $\mathrm{U}_{\mathrm{h}}=\left\{1,2, \ldots, i, \ldots, \mathrm{N}_{\mathrm{h}}\right\}$ containing $\mathrm{N}_{\mathrm{h}}$ units in hth stratum $(\mathrm{h}=1,2, \ldots, \mathrm{L})$ such that $\sum_{\mathrm{h}=1}^{\mathrm{L}} \mathrm{N}_{\mathrm{h}}=\mathrm{N}$ and let $\mathrm{W}_{\mathrm{h}}=\mathrm{N}_{\mathrm{h}} / \mathrm{N}$ be the stratum weights. A sample of size $n$, comprising of $n_{h}$ units from strata, $h$ is drawn using simple random sampling without replacement (SRSWR). Let $y_{h i}$ and 
$x_{\text {hij }}$ denote the value of $i$ th unit from hth stratum for the study variable $y$ and the $j$ th auxiliary variable $\boldsymbol{x}_{\mathfrak{j}} ; \boldsymbol{j}=1,2, \ldots, \boldsymbol{i}, \ldots, \boldsymbol{p}$, respectively. For each stratum, $h: \bar{y}_{h}=\frac{1}{n_{h}} \sum_{i=1}^{n_{h}} y_{h i}$ is the sample mean of the study variable. Assume that stratum means $\bar{X}_{h j}=\frac{1}{N_{h}} \sum_{i=1}^{N_{h}} x_{h i j}$ and the stratum variances

$$
S_{h j}^{2}=\frac{1}{N_{h}-1} \sum_{i=1}^{N_{h}}\left(x_{h i j}-\bar{X}_{h j}\right)^{2} \text {, }
$$

of all the $p$ auxiliary variables are accurately known. The purpose of the study is to propose a calibration estimator of the population mean $\bar{Y}=\sum_{h=1}^{L} W_{h} \bar{Y}_{h}$ where $\bar{Y}_{h}=\frac{1}{N_{h}} \sum_{i=1}^{N_{h}} y_{h i}$ by using the information from $p$ auxiliary variables $x_{j}$.

The stratified estimator of the population mean is given by

$$
\bar{y}_{s t}=\sum_{h=1}^{L} W_{h} \bar{y}_{h} .
$$

In the presence of $p$ auxiliary variables $x_{\mathfrak{j}} ; \boldsymbol{j}=1,2, \ldots, p$ a new calibration estimator of the population mean under stratified sampling is given by

$$
\bar{y}_{s t}^{*}=\sum_{h=1}^{L} W_{h}^{*} \bar{y}_{h},
$$

where $W_{h}^{*}$ are called the calibrated weights. The weights $W_{h}^{*}$ are so chosen such that the chi-square type distance function

$$
\sum_{j=1}^{p} \sum_{h=1}^{L} \frac{\left(W_{h}^{*}-W_{h}\right)^{2}}{W_{h} q_{h j}},
$$

is minimum, subject to the calibration constraints 


$$
\begin{gathered}
\sum_{h=1}^{L} W_{h}^{*}=1, \\
\sum_{h=1}^{L} W_{h}^{*} \bar{x}_{h j}=\sum_{h=1}^{L} W_{h} \bar{X}_{h j} ; j=1,2, \ldots, p, \\
\sum_{h=1}^{L} W_{h}^{*} d_{h} s_{h j}^{2}=\sum_{h=1}^{L} W_{h} d_{h} S_{h j}^{2} ; j=1,2, \ldots, p,
\end{gathered}
$$

where $d_{h}=\left(1 / n_{h}-1 / N_{h}\right)$ are the weights associated with the variance, $\bar{x}_{h j}=\frac{1}{n_{h}} \sum_{i=1}^{n_{h}} x_{h i j}, s_{h j}^{2}=\frac{1}{n_{h}-1} \sum_{i=1}^{n_{h}}\left(x_{h i j}-\bar{x}_{h j}\right)^{2}$ and $q_{h j}$ are suitably chosen constants to obtain different forms of the estimator. Motivated by the calibration constraint given by [14], we have proposed a similar constraint as in (6) by introducing the weights $d_{h}$.

Thus, the problem of determining the optimum calibrated weights $W_{h}^{*}$ may be formulated as an optimisation problem given below:

$$
\begin{array}{ll}
\text { Minimize: } & \sum_{h=1}^{L} \frac{\left(W_{h}^{*}-W_{h}\right)^{2}}{W_{h} Q_{h}} \\
\text { subject to } & \sum_{h=1}^{L} W_{h}^{*}=1, \\
& \sum_{h=1}^{L} W_{h}^{*} \bar{x}_{h j}=\sum_{h=1}^{L} W_{h} \bar{X}_{h j} ; j=1,2, \ldots, p, \\
& \sum_{h=1}^{L} W_{h}^{*} d_{h} s_{h j}^{2}=\sum_{h=1}^{L} W_{h} d_{h} S_{h j}^{2} ; j=1,2, \ldots, p,
\end{array}
$$

where $Q_{h}=\left(\sum_{j=1}^{p} \frac{1}{q_{h j}}\right)^{-1}$ are suitably chosen constants to obtain different forms of the estimator. 


\section{Determining the Optimum Calibrated Weights}

It can be seen that the objective function of problem (7) is convex and the constraints are linear equations, hence the Lagrange multiplier technique will yield an optimum solution. Thus we can use Lagrange multiplier technique to solve the problem (7) and determine the optimum values of $W_{h}^{*}$.

Defining $\lambda_{0}$, and $\lambda_{j}, \varphi_{j}$ for $\boldsymbol{j}=1,2, \ldots, p$, as Lagrange multipliers, the Lagrange function is given by

$$
\begin{aligned}
\phi= & \sum_{h=1}^{L} \frac{\left(W_{h}^{*}-W_{h}\right)^{2}}{W_{h} Q_{h}}-2 \lambda_{0}\left(\sum_{h=1}^{L} W_{h}^{*}-1\right) \\
& -2 \sum_{j=1}^{p} \lambda_{j}\left(\sum_{h=1}^{L} W_{h}{ }^{*} \bar{x}_{h j}-\sum_{h=1}^{L} W_{h} \bar{X}_{h j}\right) \\
& -2 \sum_{j=1}^{p} \varphi_{j}\left(\sum_{h=1}^{L} W_{h}^{*} d_{h} s_{h j}^{2}-\sum_{h=1}^{L} W_{h} d_{h} S_{h j}^{2}\right) .
\end{aligned}
$$

The necessary and sufficient conditions for solving optimum values of $W_{h}^{*}$ are

$$
\begin{gathered}
\frac{\partial \phi}{\partial W_{h}^{*}}=\frac{2\left(W_{h}^{*}-W_{h}\right)}{W_{h} Q_{h}}-2 \lambda_{0}-2 \sum_{j=1}^{p} \lambda_{j} \bar{x}_{h j}-2 \sum_{j=1}^{p} \varphi_{j} d_{h} s_{h j}^{2}=0 \\
\frac{\partial \phi}{\partial \lambda_{0}}=-2\left(\sum_{h=1}^{L} W_{h}^{*}-1\right)=0 \\
\frac{\partial \phi}{\partial \lambda_{j}}=-2\left(\sum_{h=1}^{L} W_{h}^{*} \bar{x}_{h j}-\sum_{h=1}^{L} W_{h} \bar{X}_{h j}\right)=0
\end{gathered}
$$


and

$$
\frac{\partial \phi}{\partial \varphi_{j}}=-2\left(\sum_{h=1}^{L} W_{h}^{*} d_{h} s_{h j}^{2}-\sum_{h=1}^{L} W_{h} d_{h} S_{h j}^{2}\right)=0 .
$$

From (9) we have

$$
W_{h}{ }^{*}=W_{h}+W_{h} Q_{h}\left(\lambda_{0}+\sum_{j=1}^{p} \lambda_{j} \bar{x}_{h j}+\sum_{j=1}^{p} \varphi_{j} d_{h} s_{h j}^{2}\right),
$$

where $\lambda_{0}, \lambda_{j}$ and $\varphi_{j}$ for $j=1,2, \ldots, p$ will be obtained using [4] by solving a system of nonlinear equations (10)-(12) for the given values of $W_{h}, d_{h}, \bar{x}_{h j}$ and $s_{h j}^{2}$.

\section{Numerical Illustration and Comparison Study}

In this section, we illustrate the computational details and demonstrate the performance of the proposed estimator using the tobacco data (Source: Agriculture Statistics 1999 [11]).

\subsection{Numerical Illustration}

In order to illustrate the computational details of the proposed estimator, we now describe the tobacco population. The population consists of data of $\mathrm{N}=106$ counties with three variables: area (in hectares), yield (in metric tons) and production (in metric tons). The countries were divided into $L=10$ strata and a sample of $n=40$ countries using proportional allocation was selected. 
Table 1: Population information for tobacco data.

\begin{tabular}{|l|l|l|l|l|l|l|}
\hline $\mathrm{h}$ & $\mathrm{N}_{\mathrm{h}}$ & $\mathrm{W}_{\mathrm{h}}$ & $\overline{\mathrm{X}}_{\mathrm{h} 1}$ & $\overline{\mathrm{X}}_{\mathrm{h} 2}$ & $\mathrm{~S}_{\mathrm{h} 1}^{2}$ & $\mathrm{~S}_{\mathrm{h} 2}^{2}$ \\
\hline \hline 1 & 6 & 0.05660 & 3194.5 & 1.9733 & 10899652.7 & 0.0268 \\
\hline 2 & 6 & 0.05660 & 14660.0 & 1.3883 & 584984730.0 & 0.2181 \\
\hline 3 & 8 & 0.07547 & 18309.4 & 2.5563 & 635958094.8 & 0.3470 \\
\hline 4 & 10 & 0.09434 & 14923.5 & 1.5490 & 209817189.2 & 0.2346 \\
\hline 5 & 12 & 0.11321 & 5987.8 & 1.8317 & 27842810.5 & 0.5821 \\
\hline 6 & 4 & 0.03774 & 3450.0 & 1.4700 & 5876666.7 & 0.1531 \\
\hline 7 & 30 & 0.28302 & 11682.7 & 1.1150 & 760238523.4 & 0.3439 \\
\hline 8 & 17 & 0.16038 & 145162.3 & 1.3818 & 124004506112.8 & 0.3786 \\
\hline 9 & 10 & 0.09434 & 33976.1 & 1.7210 & 8340765245.4 & 2.0183 \\
\hline 10 & 3 & 0.02830 & 1333.3 & 2.0867 & 2963333.3 & 0.9746 \\
\hline
\end{tabular}

Suppose that an estimate of average production of tobacco $(\bar{Y})$ is of interest using the two auxiliary variables $x_{1}=$ area and $x_{2}=$ yield. To determine the multivariate calibrated weights and the value of the estimate of $\bar{Y}$ in stratified sampling we use the same sample units as obtained in [11] and we assume that $\mathrm{Q}_{\mathrm{h}}=1$. The information needed for computation is summarised in Table 1 and Table 2. Substituting (13) in equations (10)-(12) and solving the system of nonlinear equations using [4] we obtain $\lambda_{0}=0.63, \lambda_{1}=2.64 \times 10^{-6}$, $\lambda_{2}=-0.50, \varphi_{1}=-4.04 \times 10^{-11}$ and $\varphi_{2}=3.12$. The optimum calibrated weights $\mathrm{W}_{\mathrm{h}}^{*}$ are obtained and presented in Column 2 of Table 3.

The calibrated weights of other estimators to be discussed in Subsection 4.2 are also presented in Columns 3, 4, 5 and 6 of Table 3 and will be later used for comparing the efficiency of the estimators.

Using (2), an estimate of the average production of tobacco using the proposed estimator is given by

$$
\overline{\mathrm{y}}_{\mathrm{st}}^{*}=\sum_{\mathrm{h}=1}^{\mathrm{L}} \mathrm{W}_{\mathrm{h}}^{*} \overline{\mathrm{y}}_{\mathrm{h}}=53585.53 .
$$


Table 2: Sample information for tobacco data.

\begin{tabular}{|l|l|l|l|l|l|l|}
\hline $\mathrm{h}$ & $\mathrm{n}_{\mathrm{h}}$ & $\overline{\mathrm{x}}_{\mathrm{h} 1}$ & $\overline{\mathrm{x}}_{\mathrm{h} 2}$ & $\mathrm{~s}_{\mathrm{h} 1}^{2}$ & $\mathrm{~s}_{\mathrm{h} 2}^{2}$ & $\overline{\mathrm{y}}_{\mathrm{h}}$ \\
\hline \hline 1 & 3 & 1304.7 & 1.9400 & 722185.3 & 0.0171 & 2592.0 \\
\hline 2 & 3 & 29075.0 & 1.3767 & 839008125 & 0.4757 & 26763.0 \\
\hline 3 & 3 & 5191.7 & 2.7933 & 74387858.3 & 0.8010 & 14559.7 \\
\hline 4 & 3 & 21700.0 & 1.4433 & 6070000.0 & 0.7362 & 29900.0 \\
\hline 5 & 4 & 6808.0 & 1.7875 & 63572981.3 & 1.0698 & 12462.5 \\
\hline 6 & 2 & 1800.0 & 1.7850 & 1620000.0 & 0.0612 & 3375.0 \\
\hline 7 & 11 & 24481.5 & 1.3209 & 1801653230.3 & 0.4824 & 38411.8 \\
\hline 8 & 6 & 294809.2 & 1.3200 & 322774101004.2 & 0.2462 & 477961.8 \\
\hline 9 & 3 & 6303.7 & 1.3267 & 59939890.3 & 0.0306 & 7480.3 \\
\hline 10 & 2 & 350.0 & 1.7650 & 125000.0 & 1.3285 & 822.5 \\
\hline
\end{tabular}

Table 3: Calibrated Weights for different methods.

\begin{tabular}{|l|l|l|l|l|l|}
\hline $\mathrm{h}$ & $\mathrm{W}_{\mathrm{h}}^{*}$ & $\mathrm{~W}_{\mathrm{h}}^{(1)}$ & $\mathrm{W}_{\mathrm{h}}^{(2)}$ & $\mathrm{W}_{\mathrm{h}}^{(3)}$ & $\mathrm{W}_{\mathrm{h}}^{(4)}$ \\
\hline \hline 1 & 0.03768 & 0.05647 & 0.05758 & 0.06450 & 0.05489 \\
\hline 2 & 0.07101 & 0.05367 & 0.07769 & 0.06075 & 0.07103 \\
\hline 3 & 0.05720 & 0.07477 & 0.08057 & 0.08531 & 0.07624 \\
\hline 4 & 0.14098 & 0.09069 & 0.12140 & 0.10292 & 0.11227 \\
\hline 5 & 0.14766 & 0.11183 & 0.12330 & 0.12753 & 0.11640 \\
\hline 6 & 0.02957 & 0.03761 & 0.03864 & 0.04296 & 0.03680 \\
\hline 7 & 0.31456 & 0.27065 & 0.36750 & 0.30688 & 0.34223 \\
\hline 8 & 0.06649 & 0.07597 & 0.06273 & 0.07046 & 0.06654 \\
\hline 9 & 0.09428 & 0.09327 & 0.20212 & 0.10638 & 0.09645 \\
\hline 10 & 0.04057 & 0.02828 & 0.01640 & 0.03231 & 0.02715 \\
\hline
\end{tabular}




\subsection{Comparison Study}

In this Subsection, using the tobacco data a comparison study is carried out on the efficiency of the following calibration estimators:

1. Singh (1998) estimator, $\bar{y}_{s t}^{(1)}=\sum_{h=1}^{L} W_{h}^{(1)} \bar{y}_{h}$ in [10].

2. Tracy (2003) estimator, $\bar{y}_{s t}^{(2)}=\sum_{h=1}^{L} W_{h}^{(2)} \bar{y}_{h}$ in [14].

3. Singh (2003) estimator, $\bar{y}_{s t}^{(3)}=\sum_{h=1}^{L} W_{h}^{(3)} \bar{y}_{h}$ in [11].

4. A univariate estimator of (2), $\bar{y}_{s t}^{(4)}=\sum_{h=1}^{L} W_{h}^{(4)} \bar{y}_{h}$ where $W_{h}^{(4)}=W_{h}+W_{h} Q_{h}\left(\lambda_{0}+\lambda_{1} \bar{x}_{h 1}+\varphi_{1} d_{h} s_{h 1}^{2}\right)$ and the auxiliary variable is $x_{1}=$ area.

5. Proposed multivariate estimator $\bar{y}_{s t}^{*}=\sum_{h=1}^{L} W_{h}^{*} \bar{y}_{h}$ in (2).

To compare the efficiency of the above estimators with respect to the stratified estimator $\bar{y}_{\text {st }}$, we compute the measure of relative efficiency (RE) as

$$
\mathrm{RE}=\frac{\hat{v}\left(\overline{\mathrm{y}}_{\mathrm{st}}\right)}{\hat{v}(\hat{\bar{Y}})} \times 100,
$$

where

$$
\hat{v}\left(\bar{y}_{s t}\right)=\sum_{h=1}^{L} W_{h}^{2} d_{h} s_{h}^{2},
$$

is the estimated variance of $\bar{y}_{\text {st }}, \hat{v}(\hat{\bar{Y}})$ is the estimated variance of a calibration estimator and $s_{h}^{2}=\frac{1}{n_{h}-1} \sum_{i=1}^{n_{h}}\left(y_{h i}-\bar{y}_{h}\right)^{2}$. The denominator $\hat{v}(\hat{\bar{Y}})$ in (15) 
Table 4: Comparison results for tobacco data.

\begin{tabular}{|l|l|l|l|}
\hline Estimator & $\hat{\bar{Y}}$ & $\hat{v}(\hat{\bar{Y}})$ & $R E$ \\
\hline \hline Stratified: $\overline{\mathrm{y}}_{\text {st }}$ & 95373.90 & 2822731121.0 & 100.0 \\
\hline Singh (1998): $\overline{\mathrm{y}}_{\text {st }}^{(1)}$ & 54329.76 & 650863727.5 & 433.7 \\
\hline Tracy $(2003): \overline{\mathrm{y}}_{\text {st }}^{(2)}$ & 54321.02 & 609889505.3 & 462.8 \\
\hline Singh (2003): $\overline{\mathrm{y}}_{\text {st }}^{(3)}$ & 54132.89 & 569781129.3 & 495.4 \\
\hline Univariate: $\overline{\mathrm{y}}_{\text {st }}^{(4)}$ & 53775.78 & 517799741.5 & 545.1 \\
\hline Proposed multivariate: $\overline{\mathrm{y}}_{\text {st }}^{*}$ & 53585.53 & 512333228.2 & 551.0 \\
\hline
\end{tabular}

is computed using the lower level calibration approach (see [11]) that is by replacing the stratum weights with the calibrated weights in equation (16).

Based on the tobacco population used in Subsection 4.1, we compare the performance of the proposed estimator based on the two auxiliary variables $\left(x_{1}=\right.$ Area and $x_{2}=$ Yield $)$ and other calibration estimators on the single auxiliary variable $\left(x_{1}=\right.$ Area $)$. It should be noted that the true average production of the tobacco crop for this population is $\bar{Y}=52444.56$. In Table 4, the Columns 2, 3 and 4 presents the estimated average production of tobacco $(\hat{\bar{Y}})$, the estimated variance $\hat{v}(\hat{\bar{Y}})$ and the relative efficiency $(R E)$ for different estimators considered.

Finally, amongst all the estimators, it was found that the proposed estimator $\bar{y}_{\text {st }}^{*}$ has the smallest estimated variance and highest RE. Thus, the study reveals that the estimator $\bar{y}_{s t}^{*}$ is the most efficient estimator of population mean in stratified sampling using the tobacco data. The gain in efficiency of the proposed estimator over the stratified estimator is $550.96 \%$.

A comparison study was also carried out using a simulated data and similar results were obtained that is the proposed estimator $\bar{y}_{\text {st }}^{*}$ has the least estimated variance and highest gain in RE and hence the most efficient estimator (see Table 5 for the results of the simulated data). The gain in efficiency of the proposed estimator over the stratified estimator is $386.85 \%$. 
Table 5: Comparison results for simulated data.

\begin{tabular}{|l|l|l|l|}
\hline Estimator & $\hat{\bar{Y}}$ & $\hat{v}(\hat{\bar{Y}})$ & $R E$ \\
\hline \hline Stratified: $\bar{y}_{\text {st }}$ & 760.951 & 45844670263.9 & 100.00 \\
\hline Singh (1998): $\bar{y}_{\text {st }}^{(1)}$ & 760.867 & 45835552384.3 & 100.02 \\
\hline Tracy $(2003): \bar{y}_{\text {st }}^{(2)}$ & 760.869 & 45745087574.3 & 100.22 \\
\hline Singh $(2003): \bar{y}_{\text {st }}^{(3)}$ & 760.868 & 45832943300.4 & 100.03 \\
\hline Univariate: $\bar{y}_{\text {st }}^{(4)}$ & 760.872 & 11870824590.0 & 386.20 \\
\hline Proposed multivariate: $\bar{y}_{\text {st }}^{*}$ & 760.882 & 11850624683.1 & 386.85 \\
\hline
\end{tabular}

\section{Conclusion}

In surveys, the statisticians are often interested to improve the precision of the survey estimates. The calibration approach is one such technique that incorporates the auxiliary information in survey sampling to improve the precision of the survey estimates.

In this paper, we considered the problem of determining the optimum calibrated weights and the optimum calibration estimator of population mean in stratified sampling, when the auxiliary information (mean and/or variance) from several variables are available. The problem is formulated as an optimisation problem that seeks minimisation of the chi-square distance function, subject to the proposed calibration constraints. The problem is then solved using the Lagrange multiplier technique. A numerical example with a real data are presented to illustrate the computational details of the proposed estimator. A comparison study with a real and a simulated data is carried out to determine the performance of the proposed estimator. The results show that the proposed estimator is the most efficient estimator of population mean in stratified sampling. Thus, it can be concluded that the precision of 
the survey estimates is further improved when multi-auxiliary information (mean and/or variance) is used as proposed.

\section{References}

[1] Jean Claude Deville and Carl Erik Särndal. Calibration estimators in survey sampling. Journal of the American statistical Association, 87(418):376-382, 1992. doi:10.1080/01621459.1992.10475217. C191

[2] Victor M Estevao and Carl Erik Särndal. Survey estimates by calibration on complex auxiliary information. International Statistical Review, 74(2):127-147, 2006. doi:110.1111/j.1751-5823.2006.tb00165.x C191

[3] Patrick J Farrell and Sarjinder Singh. Model-assisted higher-order calibration of estimators of variance. Australian \& New Zealand Journal of Statistics, 47(3):375-383, 2005. doi:10.1111/j.1467-842X.2005.00402.x C191

[4] Wolfram Research, Inc. Mathematica, Version 11.3. Champaign, IL, 2018. C196, C197

[5] Jong Min Kim, Engin A Sungur, and Tae Young Heo. Calibration approach estimators in stratified sampling. Statistics 85 probability letters, 77(1):99-103, 2007. doi:10.1016/j.spl.2006.05.015 C192

[6] Phillip S Kott. Using calibration weighting to adjust for nonresponse and coverage errors. Survey Methodology, 32(2):133, 2006. C191

[7] Dinesh K Rao. Mathematical programing in stratified random sampling. $\mathrm{PhD}$ thesis, School of Computing, Information and Mathematical Sciences, The University of the South Pacific, Fiji, February 2017. C192

[8] Dinesh K. Rao, Tokaua. Tekabu, and Mohammad G M Khan. New calibration estimators in stratified sampling. In Proceedings of 
Asia-Pacific World Congress on Computer Science and Engineering, pages 66-70. IEEE, 2016. C192

[9] Gurmindar K Singh, Dinesh K Rao, and Mohammed GM Khan. Calibration estimator of population mean in stratified random sampling. In Proceedings of Asia-Pacific World Congress on Computer Science and Engineering (APWC on CSE), pages 1-5. IEEE, 2014. C192

[10] Sarjindar Singh, Stephen Horn, and Frank Yu. Estimation of variance of general regression estimator: Higher level calibration approach. Survey Methodology, 24:41-50, 1998. C191, C199

[11] Sarjinder Singh. Advanced Sampling Theory With Applications: How Michael "Selected" Amy, volume I \& II. Kluwer Academic Publishers, Netherlands, 2003. C191, C192, C196, C197, C199, C200

[12] Sarjinder Singh. On the calibration of design weights using a displacement function. Metrika, 75(1):85-107, 2012. doi:10.1007/s00184-010-0316-6 C191

[13] Sarjinder Singh, Stephen Horn, Sadeq Chowdhury, and Frank Yu. Theory \& methods: Calibration of the estimators of variance. Australian \& New Zealand Journal of Statistics, 41(2):199-212, 1999. doi:10.1111/1467-842X.00074 C191

[14] D S Tracy, S Singh, and R Arnab. Note on calibration in stratified and double sampling. Survey Methodology, 29(1):99-104, 2003. C192, C194, C199

[15] Changbao Wu and Randy R Sitter. A model-calibration approach to using complete auxiliary information from survey data. Journal of the American Statistical Association, 96(453):185-193, 2001. doi:10.1198/016214501750333054 C191 


\section{Author addresses}

1. D. K. Rao, School of Computing, Information and Mathematical Sciences, The University of the South Pacific, Suva, FiJI. mailto:dinesh.rao@usp.ac.fj

2. M. G. M. Khan, School of Computing, Information and Mathematical Sciences, The University of the South Pacific, Suva, FiJi.

3. G. K. Singh, School of Computing, Information and Mathematical Sciences, The University of the South Pacific, Suva, FiJI. 